\title{
Indocyanine green loaded graphene oxide for high-efficient photoacoustic tumor therapy
}

\author{
Baoyun Yan and Huan Qin* \\ MOE Key Laboratory \\ of Laser Life Science $\mathcal{G}$ Institute of Laser Life Science \\ College of Biophotonics \\ South China Normal University \\ Guangzhou 510631, P.R. China \\ *qinghuan@scnu.edu.cn
}

Received 22 October 2015

Accepted 9 December 2015

Published 21 January 2016

\begin{abstract}
Photoacoustic therapy, using the photoacoustic effect of agents for selectively killing tumor cells, has shown promising for treating tumor. Utilization of high optical absorption probes can help to effectively improve the photoacoustic therapy efficiency. Herein, we report a novel highabsorption photoacoustic probe that is composed of indocyanine green (ICG) and graphene oxide (GO), entitled GO-ICG, for photoacoustic therapy. The attached ICG with narrow absorption spectral profile has strong optical absorption in the infrared region. The absorption spectrum of the GO-ICG solution reveals that the GO-ICG particles exhibited a 10-fold higher absorbance at $780 \mathrm{~nm}$ (its peak absorbance) as compared with GO. Importantly, ICG's fluorescence is quenched by GO via fluorescence resonance energy transfer. As a result, GO-ICG can high-efficiently convert the absorbed light energy to acoustic wave under pulsed laser irradiation. We further demonstrate that GO-ICG can produce stronger photoacoustic wave than the GO and ICG alone. Moreover, we conjugate this contrast agent with integrin $\alpha_{v} \beta_{3}$ mono-clonal antibody to molecularly target the U87-MG human glioblastoma cells for selective tumor cell killing. Finally, our results testify that the photoacoustic therapy efficiency of GO-ICG is higher than the existing photoacoustic therapy agent. Our work demonstrates that GO-ICG is a high-efficiency photoacoustic therapy agent. This novel photoacoustic probe is likely to be an available candidate for tumor therapy.
\end{abstract}

Keywords: Photoacoustic therapy; photoacoustic effect; tumor therapy.

${ }^{*}$ Corresponding author.

This is an Open Access article published by World Scientific Publishing Company. It is distributed under the terms of the Creative Commons Attribution 4.0 (CC-BY) License. Further distribution of this work is permitted, provided the original work is properly cited. 


\section{Introduction}

Photoacoustic therapy is an emerging tumor therapy, which uses the photoacoustic effects of probe to selectively kill tumor cells with the smallest interactions of normal cells and anti-tumor drug resistance. $^{1,4}$ In photoacoustic therapy, photoacoustic probes (or agents) are exposed to a pulsed laser, the light energy absorbed by photoacoustic probes can be converted into acoustic energy based on photoacoustic effect. ${ }^{5-14}$ Then, the magnitude of photoacoustic amplitude can generate a strong shock wave, with peak pressure of $100 \mathrm{Mpa} .{ }^{15}$ Such a strong shock wave can destroy the cell membrane, resulting in cell apoptosis. Photoacoustic therapy has several potential advantages: (1) The laser power used for photoacoustic therapy could be reduced 150-1500 times compared with other laserbased method. ${ }^{15}$ (2) Mechanical damage of cell causes smallest interactions of normal cells and antitumor drug resistance. (3) The shock waves generated from tumor-specific photoacoustic probes could be selectively treat tumors.

The key component of photoacoustic therapy is photoacoustic probes with high optical absorption. The use of photoacoustic probes with high absorption can help to improve the therapy efficiency. Numerous photoacoustic near-infrared probes such as gold nanorods, ICG and carbon nanotubes have been applied in photoacoustic therapy. ${ }^{1-3}$ However, these probes have some limitations, which limits its wide application in photoacoustic therapy. For instance, the fact that gold nanorods and gold nanoparticles are subjected to laser-induced deformation makes therapy efficiency fall. ${ }^{16-18}$ For carbon nanotubes, its absorption coefficient is relatively low in the near-infrared region. ICG with strong absorption characteristics in near-infrared range is a US FDA-approved near-infrared absorption dye. ${ }^{19,20}$ Therefore, the attentions of researchers are gradually attracted for the use of ICG in photoacoustic therapy. However, ICG is a kind of small fluorescence molecule. The absorbed light energy partly decays in radioactive channel (fluorescence). This will influence the conversion efficiency of laser to ultrasonic wave (the energy of photoacoustic signal generation only comes from the non-radiative energy). In addition, ICG is easy to be eliminated by the body, ${ }^{21}$ difficult to modify target molecules, and suffer from poor delivery to the targeted site, leading to little ICG to accumulate in local-pathological-changed tissues (such as tumor). New methods are needed to solve this problem.

In this paper, we presented ICG-loaded GO (GOICG) probes to improve photoacoustic therapy efficiency. GO with ultrahigh surface area allows many aromatic ICG molecules loading on the GO. Then, a new kind of photoacoustic therapy agent is created. Owing to narrow absorption spectral profile, the attached ICG on GO can absorb light energy with spectral selective. Importantly, ICG's fluorescence is quenched by GO via fluorescence resonance energy transfer. Under pulsed laser irradiation, GO-ICG can high-efficiently convert the absorbed light energy to acoustic waves based on the photoacoustic effect. More effective thermal expansion ultimately will improve photoacoustic therapy efficiency for killing tumor cells (Fig. 1).

GO-ICG is easy to construct. GO-ICG possesses the advantages of the GO and ICG that are biocompatibility and high optical absorption at near-infrared region. On the other hand, GO-ICG possesses several useful features such as high mechanical flexibility and without heavy metal ion-induced toxicity to living organisms that are beneficial to their application in biomedicine.

Here, GO-ICG was designed to enhance photoacoustic therapy efficiency. First, we demonstrated that at its peak absorbance at $780 \mathrm{~nm}$ the GO-ICG particles exhibited a 10-fold higher absorbance as compared with GO. We then verified that GO-ICG can produce stronger photoacoustic wave than the GO and ICG alone. We conjugate this contrast agent with integrin $\alpha_{v} \beta_{3}$ mono-clonal antibody (MAb) to molecularly target the U87-MG human glioblastoma cells for selective tumor cell killing. Finally, we measured the photoacoustic therapy efficiency of GO-ICG and GO in vitro.

\section{Materials and Methods}

\subsection{Materials}

ICG was purchased from Sigma-Aldrich Co. Ltd (Beijing, China). GO dispersion solution was obtained from Nanjing XF Nano Materials Tech Co. Ltd. (Nanjing, China). Since all the chemicals were analytical grade they were not further purified. The highpurity deionized water (resistance $>18 \mathrm{M} \Omega \cdot \mathrm{cm}$ ) is used. U87-MG human glioblastoma tumor cells were obtained from the Cell Culture Center of SunYat-sen University (Guangzhou, China). 


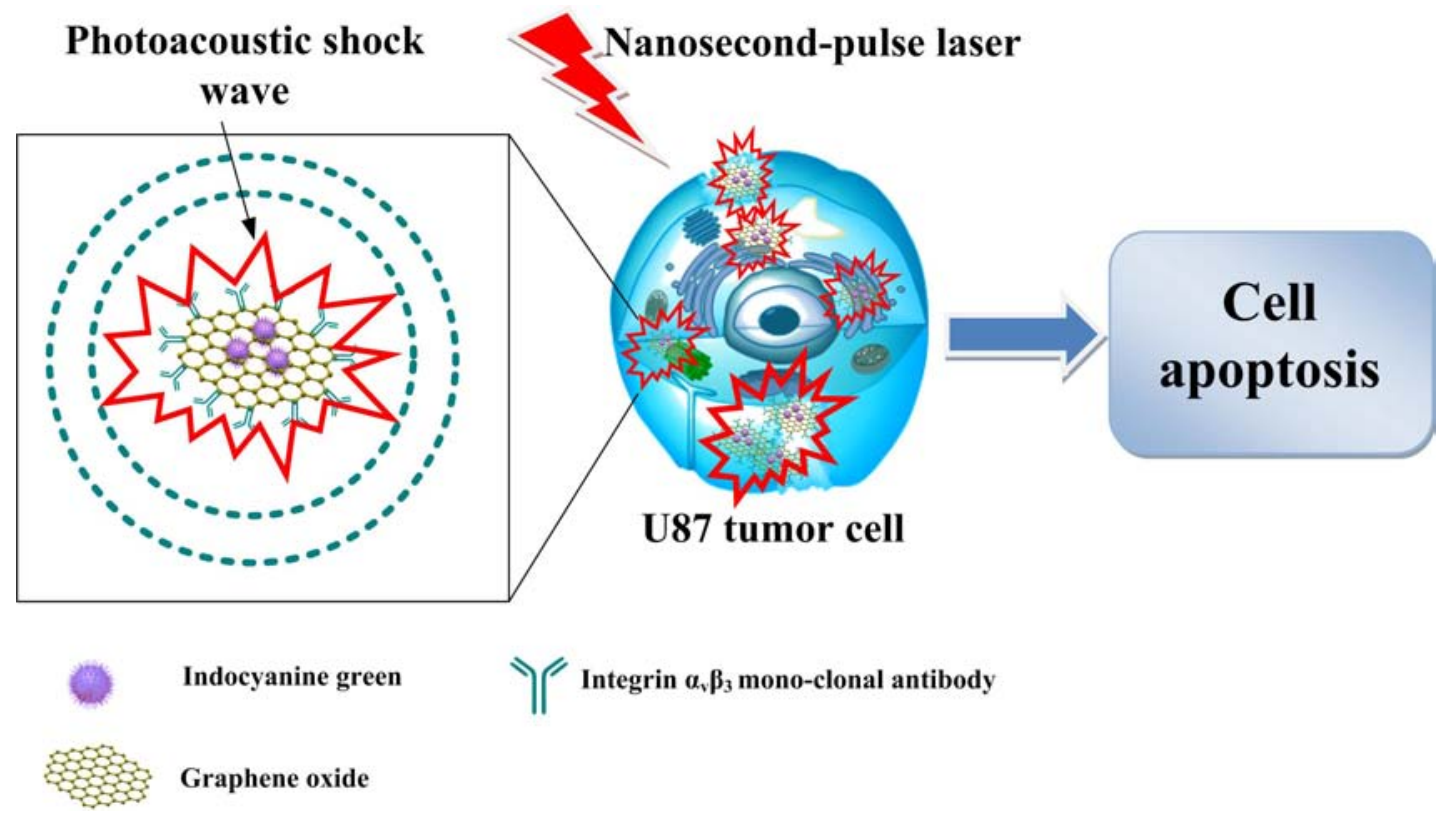

Fig. 1. The schematic diagram of photoacoustic therapy with the assistance of GO-ICG. Under pulsed laser irradiation, a strong shock wave generates from GO-ICG based on photoacoustic effect. Then, the strong shock wave could destroy the cell membrane, resulting in cell apoptosis.

\subsection{Preparation of GO-ICG}

The ICG were loaded on GO via $\pi-\pi$ interactions between $\pi$ system of ICG and GO. ICG were added into PBS of GO for preparing GO-ICG. After stirring for $2 \mathrm{~h}$, the unattached ICG were removed by being centrifuged at $13,000 \mathrm{rpm}$ for $10 \mathrm{~min}$. The GO-ICG particle suspensions contain $200 \mu \mathrm{g}$ GO and $15 \mu \mathrm{g}$ ICG per milliliter.

\subsection{Preparation of tumor-targeted $G O-I C G$ and $G O$}

Integrin $\alpha_{v} \beta_{3}$ MAb was bounded to carboxylated GO by the covalent linkage of carboxyl groups with amino groups, with EDC and NHS to accelerate the reaction. A $400 \mu \mathrm{L}$ solution of integrin $\alpha_{v} \beta_{3} \mathrm{mAb}$ at a concentration of $40 \mathrm{nM}$ in standard PBS was mixed with carboxylated GO $\left(0.1 \mathrm{mg} \mathrm{mL}^{-1}\right.$, $100 \mu \mathrm{L})$. Then $100 \mu \mathrm{L}$ of $5 \mathrm{mM}$ EDC (1-ethyl-3-(3dimethylaminopropyl) carbodiimide hydrochloride) and $100 \mu \mathrm{L}$ of $5 \mathrm{mM}$ NHS ( $N$-hydroxysuccinimide) were added to the resultant solution in succession and reacted at $4{ }^{\circ} \mathrm{C}$ overnight. Excess EDC and NHS were also removed by centrifuger and the resulting particles were redispersed in standard PBS, washed with PBS two times, redispersed in $1 \mathrm{~mL}$ PBS and then stored in a refrigerator at $4^{\circ} \mathrm{C}$.

\subsection{Characterization experiments}

Tapping mode atomic force microscopy (Agilent 5500 AFM) was used to detect the size and thickness of the GO. The absorption spectrum of GO and GO-ICG was measured by UV/visible absorption spectra (Lambda-35 UV/visible spectrophotometer, Perkin-Elmer, MA, USA). Fluorescence spectra of GO, ICG and GO-ICG were measured by spectrophotometer (LS-55 fluorescence, Perkin-Elmer).

Photoacoustic microscopy (PAM) was applied to detect photoacoustic signal intensities of GO and GO-ICG solutions. The excitation wavelength of photoacoustic signal is at $780 \mathrm{~nm}$. The repetition rate of laser system is $20 \mathrm{~Hz}$. The laser pulse width is 4-6 ns (Nd:YAG Surelight-II-20 connected to Surelite OPO Plus, Spectral tuning range 675-1000 nm, Continuum). The laser was focused by the objective lens to irradiate the target samples after expanded beam shaping. In this experiment, the laser energy density is $\sim 8 \mathrm{~mJ} / \mathrm{cm}^{2}$ at the sample surface. Ultrasonic detector with center frequency of $10 \mathrm{MHz}$ and $-6 \mathrm{~dB}$ bandwidth of $100 \%$ is purchase from Doppler Electronic Technologies Co., Ltd. (China). This ultrasonic detector is used to detect the photoacoustic signals produced from the tested samples. Subsequently, the photoacoustic signals amplified by the signal amplifier (Mini-Circuits), and then the 
photoacoustic signals were recorded by the computer through a data acquisition card with the sampling rate of $200 \mathrm{M}$ samples (National Instruments). A photoelectric detector was used to monitor stability of laser output energy, providing feedback signal to calibrate photoacoustic imaging. This photoelectric detector was purchased from Electro-Optics Technology, Inc. (Traverse City, USA).

\subsection{Cell culture}

U87-MG human glioblastoma tumor cells were cultured in Eagle's minimal essential medium (EMEM). The medium was supplemented with $10 \%$ fetal bovine serum (FBS) and 1\% penicillin-streptomycin, in $5 \% \mathrm{CO}_{2}, 95 \%$ air at $37^{\circ} \mathrm{C}$ in a humidified incubator.

\subsection{Cellular toxicity and detection}

Cytotoxicity in vitro was examined with a colorimetric tetrazolium salt-based assay by a Cell Counting Kit-8 (CCK8, Dojindo Laboratories, Kumamoto, Japan). In order to investigate cytotoxicity, U87-MG human glioblastoma tumor cells were seeded in 96-well plates. U87-MG human glioblastoma tumor cells with or without GO-Abs/ ICG incubation were irradiated by the pulse laser at energy of $<16 \mathrm{~mJ} / \mathrm{cm}^{2}$. With Infinite M200 (TECAN, Mannedorf, Switzerland), the absorbance of each well at $450 \mathrm{~nm}$ was measured. The results were expressed as the mean percentage of cell viability relative to untreated cells. Cell apoptosis was evaluated by fluorescence microscope and flow cytometry by means of Hoechst 33258 and annexin V/PI staining. The BD ApoAlert annexin V-FITC apoptosis kit was used to quantify Apoptotic cell death (Becton-Dickinson Biosciences, Mountain View, CA, USA).

\subsection{Photoacoustic therapy}

U87-MG human glioblastoma tumor cells were incubated with GO-Abs or GO-Abs/ICG. After $2 \mathrm{~h}$, to remove the photoacoustic probe in the EMEM, the samples were washed three times with PBS. Subsequently, irradiated by a pulse laser with wavelength at $780 \mathrm{~nm}(60 \mathrm{~s})$. Finally, cell viability was assessed after the U87-MG human glioblastoma tumor cells incubated in complete medium for $30 \mathrm{~min}$.

\section{Results and Discussion}

GO-ICG can be constructed using GO and ICG via $\pi-\pi$ stacking interactions. GO with ultrahigh specific surface area was utilized for loading ICG and quenching ICG fluorescence. The ICG was using for enhancing light energy absorption in near-infrared region. Atomic force microscopy was utilized to detect GO morphology. Size of GO was in the range of $100-600 \mathrm{~nm}$ (Fig. 2(a)). The absorption spectra of GO-ICG showed absorption peaks at $780 \mathrm{~nm}$ (Fig. 2(b)). GO-ICG absorption at this peak was significantly higher $(\sim$ tenfold $)$ under the same mass concentration than GO. On the other hand, we observe that GO-ICG have a weak fluorescence intensities (Fig. 2(c)). Considering the observed ICG's fluorescence quenching, we could conclude that the ICG molecules have attached to the GO forming GO-ICG nanoparticle (GO can efficiently quenching the fluorescence of dye molecules in close to $\mathrm{GO}^{4}$ ). To verify that GO-ICG can generate stronger photoacoustic signal than GO, we measured the photoacoustic signal intensities of GO and GO-ICG using a PAM system (excited at $780 \mathrm{~nm}$ ). In this experiment, the mass concentrations of GO and GO-ICG were the same. Under the same experimental conditions, the GO-ICG's photoacoustic signal intensities were stronger than GO and ICG, respectively (Fig. 2(d)). We measured photoacoustic signal intensities of GO-ICG at increasing concentrations from $10 \mu \mathrm{g} / \mathrm{mL}$ to $80 \mu \mathrm{g} /$ $\mathrm{mL}(n=3)$. The photoacoustic signal produced by the GO-ICG solution highly correlated with the GO-ICG concentration $\left(R^{2}=0.98\right.$, (Fig. 3). We could conclude that GO-ICG can produce higher photoacoustic wave than the GO due to the GOICG particles exhibited a higher absorbance. The absorbed pulse laser energy can be efficiently converted into heat by GO-ICG, causing localized volume heating, leading to rapid expansion and generation of acoustic pressure waves.

Photoacoustic therapy is a developing treatment method in tumor therapy with the help of probes as "acoustic bomb" agents. ${ }^{1-4}$ To selectively kill tumor cells, targeted GO-ICG is constructed by conjugating integrin $\alpha_{v} \beta_{3} \mathrm{mAb}$ on carboxylated $\mathrm{GO}$ (GO-Abs/ICG). Integrin $\alpha_{v} \beta_{3}$ is overexpressed on U87-MG cell cytomembranes. GO-Abs/ ICG would be an attractive candidate for photoacoustic therapy because of its high optical-acoustic conversion. To determine the photoacoustic effect of GO-ICG 


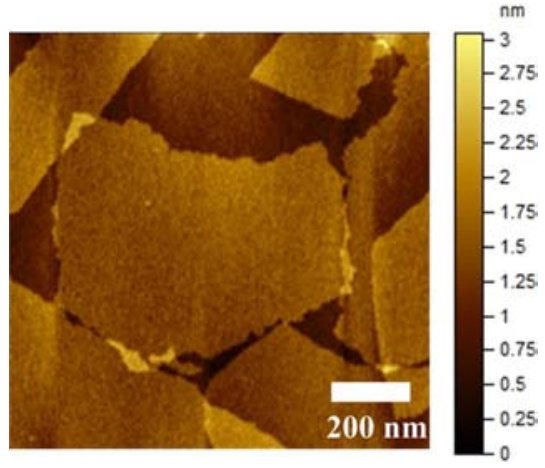

(a)

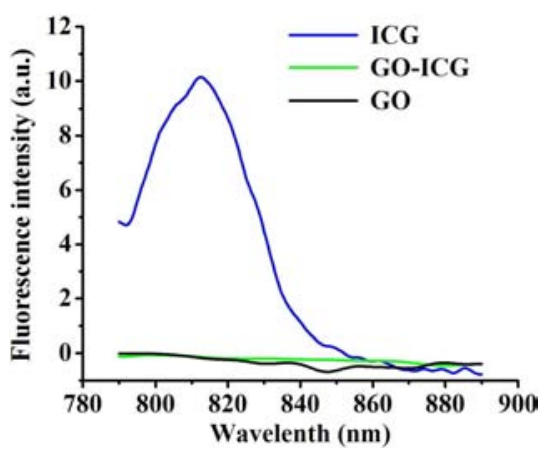

(c)

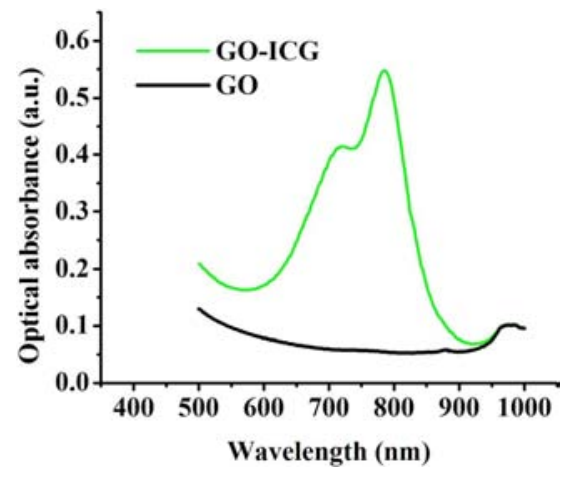

(b)

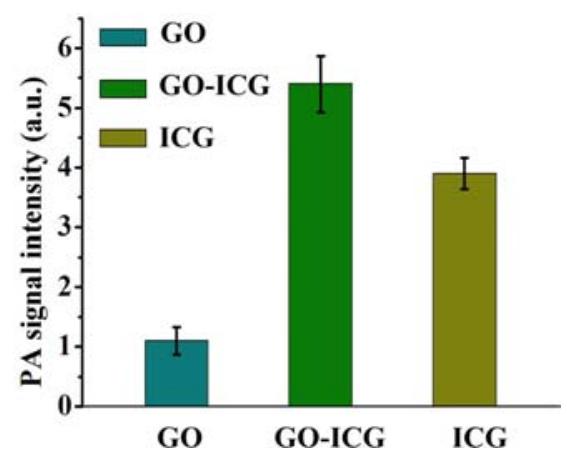

(d)

Fig. 2. Characterization of GO-ICG. (a) Atomic force microscopy image of GO. The imaging size is $1 \times 1 \mu \mathrm{m}^{2}$. (b) UV-visible absorption spectrum of GO and GO-ICG. (c) Fluorescence spectra of ICG (blue line), GO-ICG (green line) and GO (black line). (d) Photoacoustic signal intensities of GO-ICG, GO and ICG.

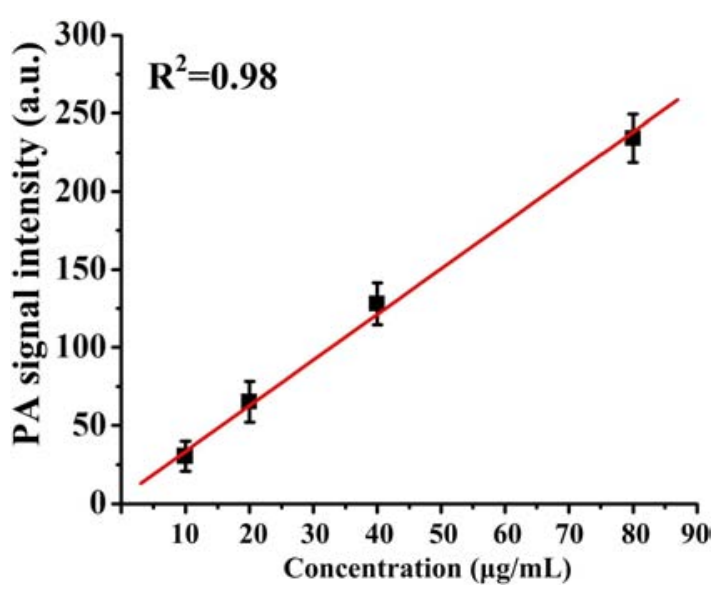

Fig. 3. The photoacoustic wave signal intensity produced by GO-ICG was linearly related to the concentration of GO-ICG $\left(R^{2}=0.98\right)$.

on cells, U87-MG cell viability after different treatments is detected. It is well known that GO-ICG cytotoxicity depended on its concentration. Cells treated with laser irradiation of $16 \mathrm{~mJ} / \mathrm{cm}^{2}$ and GO$\mathrm{Abs} / \mathrm{ICG}$ of various concentrations (ranging from
$5.37 \mu \mathrm{g} / \mathrm{mL}$ to $21.5 \mu \mathrm{g} / \mathrm{mL})$ showed significant cytotoxicity (Fig. 4(a)). To evaluate the damage effect of GO-Abs/ICG under pulsed laser excitation on U87-MG cells, Hoechst 33258 staining was performed to directly observe chromatin condensation, a sign of cell apoptosis. The results showed that with pulsed laser irradiation GO-Abs/ICG could induce U87-MG cell apoptosis (Fig. 4(b)). GO-Abs/ICG will likely to be used in photoacoustic therapy.

To further demonstrate GO-Abs/ICG being a high-effect photoacoustic therapy agent, cell apoptosis induced by different treatments was analyzed by flow cytometry with annexin-V/PI double staining assay. After incubation for $2 \mathrm{~h}$ with GOAbs/ICG (including GO-ICG with $21.5 \mu \mathrm{g} / \mathrm{mL}$ ) and GO-Abs (including GO with $21.5 \mu \mathrm{g} / \mathrm{mL}$ ), respectively, the U87-MG cells were exposed to a pulsed laser irradiation at $780 \mathrm{~nm}$. As hoped, laser excitation plus GO-Abs/ICG obviously resulted in cell death, shown by the stronger positive annexin V-FITC signal compared to that of cells treated with laser irradiation plus GO-Abs (Fig. 5). For 


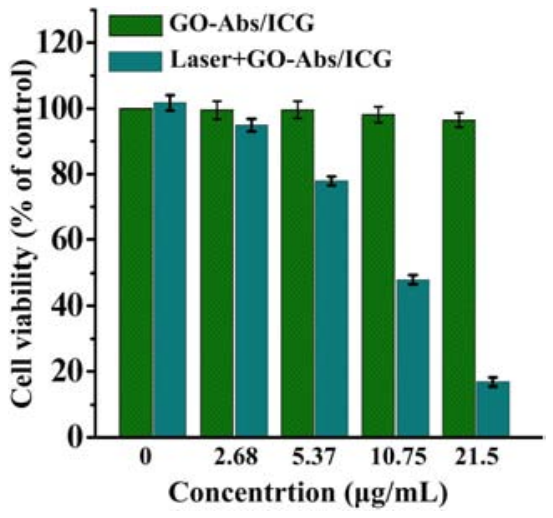

(a)

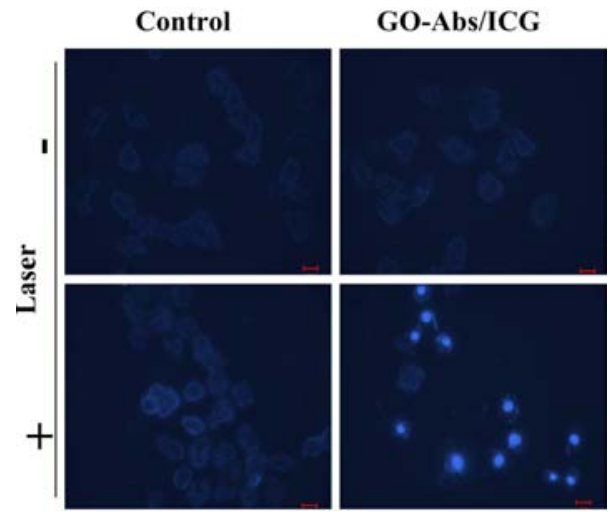

(b)

Fig. 4. The photoacoustic therapeutic effect of GO-Abs/ICG on U87-MG cell. (a) Cell toxicity test under the different doses of GO-Abs/ICG $(n=4)$. The concentrations of GO-Abs/ICG are ranging from $2.68 \mu \mathrm{g} / \mathrm{mL}$ to $21.5 \mu \mathrm{g} / \mathrm{mL}$. The laser energy density using in photoacoustic therapy is $16 \mathrm{~mJ} / \mathrm{cm}^{2}$. (b) After photoacoustic therapy, U87-MG cells apoptosis are evaluated by Hoechst 33258 morphological examination.

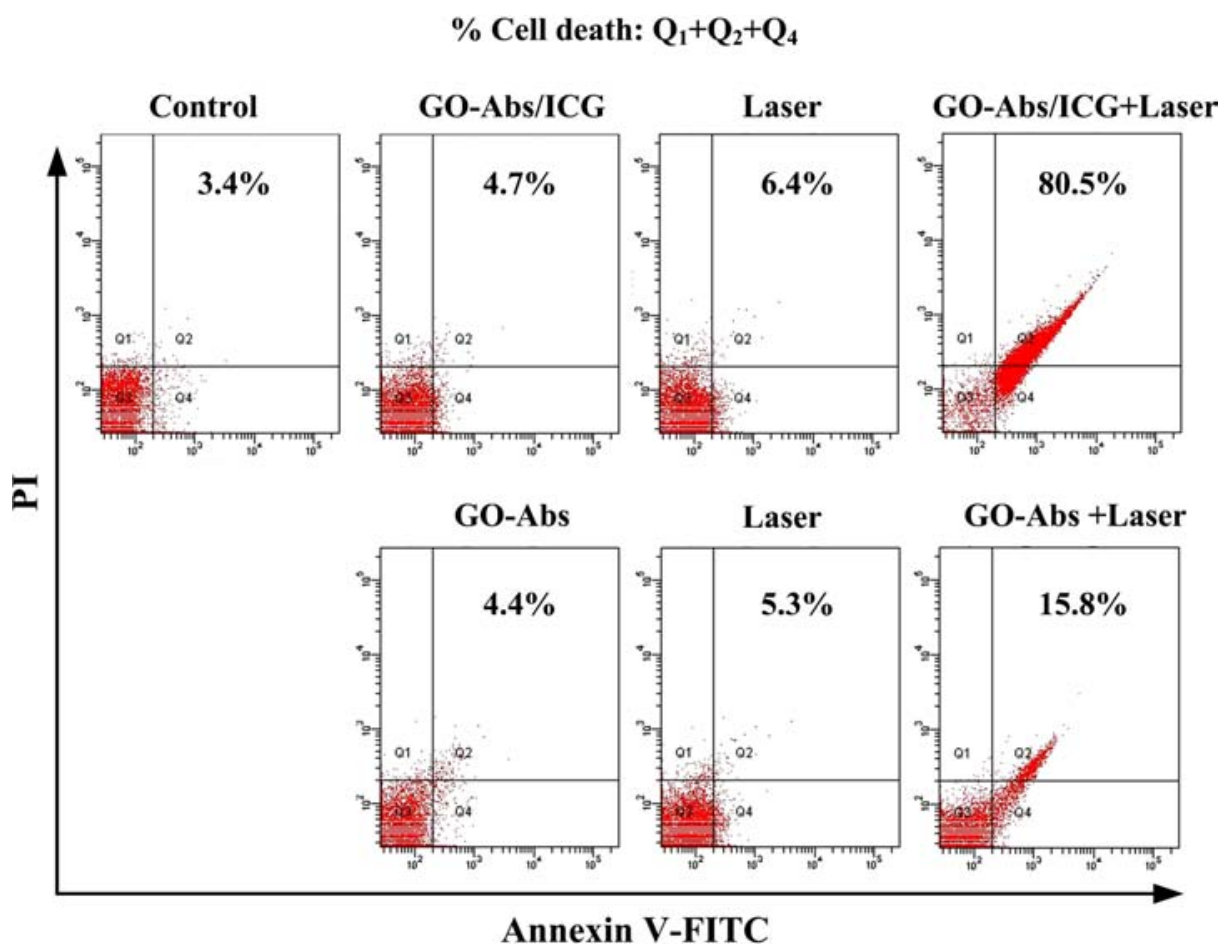

Fig. 5. The comparison of photoacoustic therapy efficiency between GO-Abs/ICG and GO-Abs at the different treatments. Analysis of apoptosis in differently treated U87-MG cells was performed by flow cytometry with annexin-V/PI double staining assay.

GO-Abs/ICG (80.5\%), the efficiency of killing tumor cells was higher than that of GO-Abs (15.8\%). That the strong laser-induced wave from GO-Abs/ICG can effectively induce U87-MG cell apoptosis and GO-Abs/ICG has high efficiency at killing tumor cells making it convenient to use in photoacoustic therapy.
We have synthesized, characterized, and demonstrated the application of ICG-enhanced GO as a high efficiency photoacoustic therapy agents. The optical absorbance spectrum of the new GO-ICG nanoparticles reveals that at its peak absorbance at $780 \mathrm{~nm}$ the GO-ICG particles exhibited a 10-fold higher absorbance as compared with plain GO. 
GO-ICG can produce stronger photoacoustic wave than the GO and ICG alone, respectively. Moreover, integrin $\alpha_{v} \beta_{3}$ MAbs are conjugated to GO-ICG to molecularly target the U87-MG human glioblastoma tumor cells for selective tumor cell killing. The photoacoustic therapy efficiency of GO-ICG was found to be higher than that commonly used photoacoustic therapy agent. In vivo GO-Abs/ICG photoacoustic therapy effect in tumor will be study in next step.

Photoacoustic imaging is a kind of non-invasive and non-ionizing new biomedical imaging method developed in recent years. ${ }^{4,5}$ When pulse laser irradiation to biological tissue, optical absorber in tissue can be produced ultrasonic signal based on photoacoustic effect. Photoacoustic signals from the biological tissue carry the light absorption characteristics of absorber in tissue. Absorber distribution in the tissue can be reconstructed through detection of photoacoustic signal. Photoacoustic imaging overcomes the optical diffusion limit by integrating optical excitation with ultrasonic detection based on the photoacoustic effect, providing deeper tissue imaging with high spatial resolution. Both photoacoustic therapy and photoacoustic imaging are based on the photoacoustic effect of moleculars or nanoprobes. The GO-ICG developed here not only could be applied in photoacoustic therapy, but also could apply to photoacoustic imaging. Photoacoustic imaging allows deep-seated tumors imaging with higher spatial resolution compared to pure optical techniques (such as fluorescence imaging and optical coherence tomography). According to common point of photoacoustic imaging and photoacoustic therapy, an integrated diagnosis and therapy protocol could be instituted: Using photoacoustic imaging to detect the deep location of the tumor which could provide accurate spatial location information of tumor. Next, with the guidance of photoacoustic imaging, photoacoustic therapy can conduct regional selective treatment of tumor. GO with ultrahigh specific surface area also allows loading antitumor drugs on GO and delivering the drugs to the targeted location (such as tumor lesion area). This novel nanoplatform may be applied in photoacoustic imaging, photoacoustic therapy and chemotherapy.

\section{Conclusion}

In summary, we have presented a novel highabsorption photoacoustic probe (GO-ICG) that is composed of ICG and GO for photoacoustic therapy.
We successfully demonstrate that this probe can selectively enhance photoacoustic therapy efficiency in vitro. This new photoacoustic probe has the potential applications for photoacoustic therapy in vivo.

\section{Acknowledgments}

This research is supported by the National Basic Research Program of China (2011CB910402; 2010CB732602), the National Natural Science Foundation of China (61361160414; 61331001; 81127004), and the Guangdong Natural Science Foundation (S2013020012646).

\section{References}

1. F. Zhou, S. Wu, Y. Yuan, W. R. Chen, D. Xing, "Mitochondria-targeting photoacoustic therapy using single-walled carbon nanotubes," Small $\mathbf{8}$, 1543-1550 (2012).

2. J. Zhong, S. Yang, X. Zheng, T. Zhou, D. Xing, "In vivo photoacoustic therapy with tumor-targeted indocyanine green-containing nanoparticles," Nanomedicine 8, 903-919 (2013).

3. J. Zhong, L. Wen, S. Yang, L. Xiang, Q. Chen, D. Xing, "Imaging-guided high-efficient photoacoustic tumor therapy with targeting gold nanorods," Nanomed.: NBM 11, 1499-1509 (2015).

4. H. Qin, T. Zhou, S. Yang, D. Xing, "Fluorescence quenching nanoprobes dedicated to in vivo photoacoustic imaging and high-efficient tumor therapy in deep-seated tissue," Small 11, 2675-2686 (2015).

5. S. Yang, D. Xing, Q. Zhou, L. Xiang, Y. Lao, "Functional imaging of cerebrovascular activities in small animals using high-resolution photoacoustic tomography," Med. Phys. 34, 3294-3301 (2007).

6. Y. Zhao, S. Yang, C. Chen, D. Xing, "Simultaneous optical absorption and viscoelasticity imaging based on photoacoustic lock-in measurement," Opt. Lett. 39, 2565-2568 (2014).

7. J. Zhang, S. Yang, X. Ji, Q. Zhou, D. Xing, "Characterization of lipid-rich aortic plaques by intravascular photoacoustic tomography: Ex vivo and in vivo validation in a rabbit atherosclerosis model with histologic correlation," J. Am. Coll. Cardiol. 64, 385-390 (2014).

8. L. V. Wang, S. Hu, "Photoacoustic tomography: In vivo imaging from organelles to organs," Science 335, 1458-1462 (2012).

9. Z. Ji, Y. Fu, S. Yang, "Microwave-induced thermoacoustic imaging for early breast cancer detection," J. Innov. Opt. Heal. Sci. 6, 1350001 (2013). 
10. Y. Zhao, S. Yang, "Photoacoustic viscoelasticity imaging of biological tissues with intensity-modulated continuous-wave laser," J. Innov. Opt. Heal. Sci. 6, 1350033 (2013).

11. J. Zhong, S. Yang, "Contrast-enhanced photoacoustic imaging using indocyanine green-containing nanoparticles," J. Innov. Opt. Heal. Sci 7, 1350029 (2014).

12. H. Qin, T. Zhou, S. Yang, Q. Chen, D. Xing, "Gadolinium (III)-gold nanorods for MRI and photoacoustic imaging dual-modality detection of macrophages in atherosclerotic inflammation," Nanomedicine 8, 1611-1624 (2013).

13. H. Qin, S. Yang, D. Xing, "Microwave-induced thermoacoustic computed tomography with a clinical contrast agent of $\mathrm{NMG}_{2}[\mathrm{Gd}$ (DTPA)]," Appl. Phys. Lett. 100, 033701 (2012).

14. L. Nie, P. Huang, W. Li, X. Yan, A. Jin, Z. Wang, $\mathrm{X}$. Chen, "Early-stage imaging of nanocarrierenhanced chemotherapy response in living subjects by scalable photoacoustic microscopy," ACS Nano 8, 12141-12150 (2014).

15. B. Kang, Y. Dai, S. Chang, D. Chen. "Explosion of single-walled carbon nanotubes in suspension induced by a large photoacoustic effect," Carbon $\mathbf{4 6}$, 974-981 (2008).

16. S. Link, C. Burda, M. B. Mohamed, B. Nikoobakht, M. A. El-Sayed, "Laser photothermal melting and fragmentation of gold nanorods: Energy and laser pulse-width dependence," J. Phys. Chem. A. 103, 1165 (1999).

17. S. Link, B. Burda, B. Nikoobakht, M. A. El-Sayed, "Laser-induced shape changes of colloidal gold nanorods using femtosecond and nanosecond laser pulses," J. Phys. Chem. B 104, 6152 (2000).

18. C. M. Aguirre, C. E. Moran, J. F. Young, N. J. Halas, "Laser-induced reshaping of metallodielectric nanoshells under femtosecond and nanosecond plasmon resonant illumination," J. Phys. Chem. B 108, 7040 (2004).

19. M. Ogawa, N. Kosaka, P. L. Choyke, H. Kobayashi, "In vivo molecular imaging of cancer with a quenching near-infrared fluorescent probe using conjugates of monoclonal antibodies and indocyanine green," Cancer Res. 69, 1268-1272 (2009).

20. J. Yu, D. Javier, M. A. Yaseen, "Self-assembly synthesis, tumor cell targeting, and photothermal capabilities of antibody-coated indocyanine green nanocapsules," J. Am. Chem. Soc. 132, 1929-1938 (2010).

21. A. de la Zerda, S. Bodapati, R. Teed, S. Y. May, T. S. M. Abakman, Z. Liu, B. T. Khuri-Yakub, X. Chen, H. Dai, S. S. Gambhir, "Family of enhanced photoacoustic imaging agents for high-sensitivity and multiplexing studies in living mice," ACS Nano 6, 4694 (2012). 